\title{
Evangelisierung und Kommunikationsmittel nach dem Puebla-Dokument
}

\author{
von Horst Rzepkowski
}

Das Verhältnis von Presse, Funk und Fernsehen und der Dritten Generalversammlung der Lateinamerikanischen Bischöfe in Puebla de Los Angeles vom 27. Januar bis zum 13. Februar 1979 kann mit zwei Worten umschrieben werden: Mißtrauen und Mißverständnis. Noch nie hatten sich so viele Journalisten zu einer kirchlichen Konferenz angesagt, aber auch nnch nie war der Kontakt zwischen den Medien und der Konferenz so schwierig und mager. Es herrschten Ängstlichkeit, Unvermögen und Unkenntnis ${ }^{1}$.

Man machte in diesem Zusamenhang darauf aufmerksam, daß das schwierige Verhältnis zu den Massenmedien ein spezifisch kirchliches Problem sei². So ist die Frage nach der Stellung der Puebla-Konferenz in den Kommunikationsmitteln durchaus berechtigt.

\section{Die Aussagen von Puebla zu den Massenmedien}

Das Dokument der Puebla-Konferenz behandelt die Fragen der Kommunikationsmittel mehrmals. In den verschiedenen Abschnitten macht man auf den Gebrauch und die Hilfe durch die sozialen Kommunikationsmittel aufmerksam. Bei der Auflistung der pastoralen Situation im kulturellen-sozialen Kontext werden die sozialen Kommunikationsmittel im Zusammenhang mit der Frage der Erziehung behandelt und ihre Funktion als Kulturübermittler hervorgehoben. Es wird gesagt, durch die sozialen Kommunikationsmedien werde die kulturelle Welt stark beeinflußt. Weiterhin heißt es: „Durch sie können die politischen, ideologischen und wirtschaftlichen Machtgruppen gleichzeitig in die Umwelt und die Lebensart unseres Volkes eindringen. Die Mächtigen manipulieren die Nachrichten. Dies geschieht insbesondere durch die Werbung, die falsche Erwartungen weckt, Scheinbedürfnisse erzeugt und in vielen Fällen den Grundwerten unserer lateinamerikanischen Kultur und dem Evangelium widersprechen. Der Mißbrauch der Freiheit in den Medien führt dazu, daß man bis in die Privatsphäre der Menschen eindringt, die meist schutzlos sind. Sie durchdringen alle Bereiche des Lebens (Wohnung, Arbeitsplatz, Freizeit und Straße) und wirken 24 Stunden. Außerdem führen sie zu kulturellen Änderungen, die eine neue Sprache schaffen."

Die Bedrohung durch Rundfunk, Fernsehen und Presse wird wiederholt angesprochen. Im Zusammenhang mit der Familie wird gesagt, daß der Einfluß der Medien für die Ehe und Familie bedrohlich ist. Durch die Darstellung der ehelichen Untreue, der Scheidung und der Vergötzung der Geschlechtlichkeit werden die Grundlagen der Familie angetastet. Der unangemessene Gebrauch von Rundfunk und Fernsehen kann zur Gefahr für die religiösen Praktiken in der Familie werdens. Für die Jugend wird festgestellt, daß ein Großteil ihrer Freizeit von den sozialen Kommunikationsmitteln beansprucht und gestaltet wird ${ }^{6}$. Man fordert von der Jugend einen kritischen Sinn im Gebrauch der Massenmedien, die nicht nur dem Einfluß der Kirche entzogen sind, sondern in den meisten Staaten „kulturlose und kulturschädliche” Programme senden. Meist auch von Parteien und Ideologien manipuliert werden ${ }^{7}$.

Prof. P. Dr. Horst Rzepkowski svd ist Dozent für Missionswissenschaft an der philosophischtheologischen Hochschule St. Augustin. 
Gegen Ende des Dokumentes wendet man sich an alle Verantwortlichen für die Massenmedien. Man solle einen Kodex erstellen und einhalten, der durch die Forderung der Information und Kommunikation bestimmt ist. Die Massenmedien sind in sich betrachtet neutral, aber sie werden durch den Gebrauch in ihrem Wert bestimmt. Sie sollen die objektive Wahrheit und Bilḍung übermitteln und zu einer genügenden Einsicht in die Wirklichkeit führen ${ }^{8}$.

Man fordert die Laien auf, sich mit besonderer Aufmerksamkeit den Massenmedien zu widmen ${ }^{9}$. Muß aber im gleichen Zusammenhang bekennen, daß das Engagement der Laien in den Bereichen der Presse, des Rundfunks und Fernsehens fast ganz fehlt ${ }^{10}$. In der Beschreibung der Situation der Liturgie in Lateinamerika wird der Priestermangel angesprochen. Es wird gesagt, man habe mit den Wortgottesdiensten gute Erfahrungen gemacht. Für die Feier dieser Wortgottesdienste ist von einer entscheidenden Dringlichkeit, sich der Massenmedien, des Radios und des Fernsehens zu bedienen, um möglichst alle zu erreichen ${ }^{11}$. Sind diese Ausführungen über die sozialen Kommunikationsmittel mehr zufällig, so wird in dem Abschnitt über die Verbreitung der Frohen Botschaft neben der Liturgie, dem Zeugnis, der Katechese und Erziehung die Frage der Massenmedien ausführlich angesprochen.

Dem Abschnitt wird in Thesenform eine Beschreibung des Zusammenhanges von Evangelisierung und Kommunikation vorangestellt. Die Evangelisierung ist Kommunikation, und dieser Tatsache muß man in allen ihren Aspekten Rechnung tragen bei der Übermittlung der Frohbotschaft. Die Kommunikation ist ein lebendiger sozialer Akt, der mit dem Menschen selbst gegeben ist. Durch technische Erfindungen in der modernen Zeit hat sie an Bedeutung gewonnen. Für die Evangelisierung darf man auf diese Mittel nicht verzichten ${ }^{12}$.

Ein längerer Abschnitt beschreibt die lateinamerikanische Wirklichkeit im Bereich der Massenmedien. Er stellt die Bedeutung der sozialen Kommunikationsmittel für die Einzelperson und den gesamten gesellschaftlichen Raum dar. Es wird aber auch der Mißbrauch von Presse, Rundfunk und Fernsehen durch politische und wirtschaftliche Machtgruppen angeprangert. Vielfach schaffen diese durch die Massenmedien Abhängigkeiten und Unterdrückung, verherrlichen Gewalt und Sex und verletzen die Rechte der Einzelmenschen. Sie unterhöhlen den Respekt vor der Person und die Grundlage der Familie. Es wird von der Presse eine objektive Übermittlung der Nachrichten erwartet. Die Monopolisierung der Informationen durch die Regierung oder durch Privatinteressen werden verurteilt, weil so der Mißbrauch der Massenmedien wächst. Auch beklagt man, daß die Nachrichten in vielen Staaten durch ausländische Mächte bestimmt werden. Die vielen ausländischen Programme zerstören und überfremden vielfach einheimische kulturelle Werte. Es wird der zerstörerische Einfluß der Massenmedien auf die Familie beklagt. Die Massenmedien werden vielfach in Propagandavehikel für den Materialismus verkehrt. Man weckt in der Bevölkerung falsche Erwartungen und Scheinbedürfnisse, was schwerwiegende Folgen nach sich zieht ${ }^{13}$.

Die anschließende Beschreibung der kirchlichen Situation beginnt mit der Feststellung, $\mathrm{da} ß$ die lateinamerikanische Kirche sich der Bedeutung der Massenmedien voll bewußt ist. Die Kirche hat in zahlreichen Dokumenten die Bedeutung der sozialen Kommunikationsmittel herausgestellt.

Dennoch muß man einen langen Katalog von Versäumnissen aufführen. Es fehlen die notwendigen Verbindungen zwischen den Medien und der Kirche, so werden wichtige Möglichkeiten nicht genutzt. Der Einsatz und Einfluß der eigenen Medien der Kirche 
ist zu schwach. Im lateinamerikanischen Bereich gibt es keine Stelle und Organisation, die sich der Schulung und Unterweisung der Gläubigen im Gebrauch und in der aktiven Kritik der Medien widmet. So sind die Gläubigen dem „Bombardement durch die Massenmedien” ausgesetzt. Sie sind dem Einfluß von Ideologien ausgesetzt. Darum müssen Kurse angeboten werden, die die Funktion der Massenmedien innerhalb der Evangelisierung aufweisen, wobei man aber auch nicht die Eigentümer und Techniker der entsprechenden Einrichtungen der Massenmedien vernachlässigen darf. Durch die schnelle Entwicklung der sozialen Kommunikationsmittel wächst auch das Material, das in der Evangelisierung gebraucht werden kann und den Pastoralträgern zur Verfügung steht und die Möglichkeiten des Kontaktes und des Dialogs vervielfacht.

Zwar hat die Kirche in den letzten Jahren kraftvolle Anstrengungen gemacht, um eine bessere innerkirchliche Kommunikation zu fördern. Aber dennoch entsprechen diese in vielen Fällen nicht den Anforderungen der Wirklichkeit und des Augenblicks. In der öffentlichen Meinung in der Kirche werden diese Anstrengungen nur als sporadisch und als völlig unzureichend gewertet und haben letztlich nur einen geringen Einfluß auf die Gesamtheit der kirchlichen Gemeinschaft ${ }^{14}$.

Für die praktischen Folgerungen werden einige Kriterien aufgestellt. Die Kommunikation ist in den Rahmen der Gesamtpastoral (pastoral de conjunto) integriert. Innerhalb der Verwirklichung der Folgerungen hat die Ausbildung in den Kommunikationsmitteln Vorrang. Dieses nicht nur für das Kirchenvolk, sondern auch für alle Pastoralträger auf allen Ebenen. Die Meinungsfreiheit und die Rückgebundenheit der Massenmedien an die Gesellschaft und ihre Funktion in der Gesellschaft müssen beachtet werden. Ein entsprechender Verhaltenskodex sollte sich an den Prinzipien der Pastoralinstruktion „Communio et Progressio" orientieren ${ }^{15}$. Für die konkrete Arbeit werden in dem folgenden Abschnitt eine ganze Reihe Vorschläge gemacht und gezielte Forderungen aufgestellt. Die Träger der Pastoral müssen eine gediegene Kenntnis von der Wirkweise der Kommunikationsmittel haben, damit diese entsprechend in die Pastoral einbezogen werden.

Eine ständige Weiterführung der Ausbildung wird die Pastoral aktualisieren. Dafür bedarf es aber eines Zentrums auf nationaler und Diözesanebene. Dringend und vorrangig ist die Ausbildung im Bereich der sozialen Kommunikationsmittel für die Träger der Evangelisierung. Die Kandidaten für das Priestertum und Ordensleben bedürfen notwendigerweise einer Ausbildung in diesen Fragen, und diese soll in den Studienplan integriert werden. Prister, Ordensleute und Träger der Pastoral, die für die Sozialpastoral in der Diözese und auf nationaler Ebene verantwortlich sind, sollen eine fortgesetzte Einführung und Schulung erhalten. Besonders brennend sind diese Fragen für die Fachleute, sie bedürfen einer angemessenen Schulung über die religiöse Information.

Für die Kirche bedeutet das, daß sie innerhalb der liturgischen Normen und Vorschriften für eine entsprechende Einführung in die Liturgie sorgt, Symbole und Formen entwickelt, die die Liturgie und den Bezug zu Gott verständlicher und die Teilnahme an der Liturgie vollziehbarer machen.

Die Bevölkerung muß zu einer aktiven Kritik gegenüber den Medien und ihrer Manipulation geführt werden. Dafür sollte sie aber auch die kirchlichen Organisationen kennen, die sich dieser Aufgabe stellen. 
Die kirchliche Sprache muß konkreter, unmittelbarer, klarer und sorgfältiger werden. Die Sprache soll der Mentalität und der Frömmigkeit des Volkes angepaßt sein. Durch die audio-visuellen Mittel soll man dem heutigen Menschen die entsprechenden Inhalte nahebringen.

Die Kirche soll sich der vorhandenen Mittel und Wege bedienen, daneben muß sie aber nach eigenen Möglichkeiten der Verwirklichung in den Bereichen der Information und Nachrichtenvermittlung suchen, um mit der Welt von heute in einen Dialog eintreten zu können. Besondere Gebiete der kirchlichen Wirksamkeit sind Armut, Unterdrückung, Unrecht und Marginalisierung. Die Kirche muß in ihren Bereichen und mit ihren Kommunikationsmitteln die Menschenrechte verteidigen. Sie muß Stimme der Unterdrückten werden.

Sie muß die Rechte der Information im Rahmen der ethischen Bindungen und Verpflichtungen verteidigen, das heißt die Achtung vor dem Privatbereich der Person und vor der Wahrheit. Diese Prinzipien gelten auch innerhalb der Kirche, müssen aber mit Demut gegenüber ungerechten Forderungen verteidigt werden ${ }^{16}$.

Die in wesentlichen Linien nachgezeichnete Haltung der lateinamerikanischen Bischofskonferenz zu den Massenmedien stützt sich auf die Pastoralinstruktion „Communio et Progressio ${ }^{17}$ und „Evangelii nuntiandi” ${ }^{18}$. Die Hinweise und Forderungen sind auch vielleicht gar nicht spezifisch für die lateinamerikanische Wirklichkeit. Dennoch sind die Hinweise auf die Verteidigung der Menschenrechte und Personwürde durch die Erfahrungen des Kontinents geprägt. Aber entscheidend an diesen Texten ist, daß Evangelisierung und Kommunikation in ihrem Bezug gesehen werden. Bedeutend für diesen Zusammenhang ist weiter, daß man entschieden für die Integrierung der Kommunikationsmittel in die Pastoral eintritt. Die Massenmedien sind nicht eine Sorge, ein Kriterium der kirchlichen Aufmerksamkeit neben anderen, sondern werden in eine umfassende Pastoralplanung einbezogen. Die entsprechenden Hinweise für eine wache Kritik innerhalb des Kirchenvolkes und die fortschreitende Ausbildung aller Verantwortlichen sind wichtige Zielpunkte für die Zukunft der kirchlichen Entwicklung in Lateinamerika.

Die Kommunikation ist nicht nur eine Frage der missionarischen Methode oder des Gebrauchs der Massenmedien für die Evangelisierung und Pastoral. Sie steht immer in einem theologischen Zusammenhang mit der Heilsgeschichte in Christus, der Hinwendung Gottes zur Welt. „Kommunikation in der Mission hat nur insoweit Sinn, als sie Teil der Kommunikation Gottes mit der Welt ist, dessen, was Gott getan hat und noch tut." 19

\section{Versuch einer Einordnung}

In einem Kurzkommentar zum Hauptabschnitt über die Massenmedien im Abschlußdokument von Puebla hebt Luciano M. Metzinger zwei Punkte als entscheidend hervor. Es wird der innere Bezug von sozialer Kommunikation und Evangelisierung klar erkannt und angesprochen. Zweitens wird die Bedeutung der sozialen Kommunikationsmittel im gegenwärtigen sozio-kulturellen Prozeß hervorgehoben. Mit der ersten Feststellung befindet man sich auf der Linie der großen kirchlichen Verlautbarungen zu dieser Thematik. Diese hatten auf den Bezug und die Notwendigkeit der Massenmedien bei der Evangelisierung aufmerksam gemacht. Die daraus resultierenden Folgerungen für die direkte und konkrete Pastoral sind auch wiederholt aufgestellt worden, 
aber die Integration der Kommunikation in den Gesamtpastoralplan ist ein entscheidender Schritt mehr. Die Aussagen über den sozio-kulturellen Impuls der Massenmedien verlangen eine größere Aufmerksamkeit und eine direkte aktive christliche Mitarbeit. Besonders dieses zweite Faktum wertet Metzinger als etwas entscheidend Neues innerhalb eines kirchlichen Dokumentes ${ }^{20}$. Dieser Kommentar und die Hinweise im Puebla-Dokument fordern zu einem Vergleich mit den großen kirchlichen Verlautbarungen zu den Massenmedien heraus. Aber ein solcher Vergleich würde dem Anliegen der lateinamerikanischen Bischöfe wohl nicht gerecht. Die römischen Dokumente „Inter Mirifica” und „Communio et Progressio" bauen auf den Aussagen des Zweiten Vatikanischen Konzils über die sozialen Kommunikationsmittel auf und entfalten die Ansätze weiter. Ihr Blickpunkt und ihre Zielrichtung ist anders. In Puebla war der theologische Ansatz die Frage nach der Evangelisierung und der Pastoral.

Von daher bietet sich ein Vergleich mit den Aussagen über die Massenmedien und die Evangelisierung in „Evangelii nuntiandi” an ${ }^{21}$. Wie die Bischofskonferenz in Medellin (1965) die Aussagen und Erwartungen des Zweiten Vatikanischen Konzils an die lateinamerikanische Wirklichkeit heranzutragen versuchte, so wollte man in Puebla die Forderungen des Missionsrundschreibens „Evangelii nuntiandi” für die lateinamerikanische Kirche ausformulieren ${ }^{22}$. Eine solche Parallele ergibt sich aus der verwandten Zielsetzung beider Dokumente.

In beiden Dokumenten sind die Kommunikationsmittel in den Kontext der Vermittlung der Botschaft eingebettet. Sie haben für die Verkündigung entscheidende Bedeutung. In „Evangelii nuntiandi” wird gesagt: „Die Kirche würde vor ihrem Herrn schuldig, wenn sie nicht diese machtvollen Mittel nützte, die der menschliche Verstand immer weiter vervollkommnet."22 Die Dringlichkeit zum Gebrauch wird also in beiden Papieren herausgestellt. Aber das Puebla-Dokument hebt sich in wichtigen Punkten von „Evangelii nuntiandi” ab. Es bietet eine theologische Integrierung der Kommunikation in die Evangelisierung. Es versteht den Prozeß der Evangelisierung als einen Kommunikationsvorgang ${ }^{23}$. Die Forderungen nach der entsprechenden Ausbildung des Klerus werden hier ausdrücklich gestellt; dies sind keine neuen Postulate; sie sind aber in ihrer Akzentsetzung zu beachten.

Was neu an den Puebla-Aussagen ist, wird durch eine Gegenüberstellung mit dem Vorbereitungspapier zur Dritten Lateinamerikanischen Bischofskonferenz noch deutlicher. Auch dieses Dokument hatte in dem Abschnitt über die Prioritäten innerhalb der Evangelisierung einen eigenen Beitrag über die Massenmedien. Es wurden die gegenwärtige Situation beschrieben und aus den Notwendigkeiten und den vorhandenen Möglichkeiten Forderungen für die Kirche gezogen. Es wurde gegen Ende dieses Abschnitts auch gefordert, da $\beta$ die Pastoralagenten eine entsprechende Kenntnis der Massenmedien besitzen sollen ${ }^{24}$. Es wurde aber nicht die Kommunikation als ein Evangelisierungsprozeß $\$$ verstanden, und die Massenmedien wurden nicht in den Pastoralplan einbezogen.

Wenn auch das Vorbereitungspapier auf der Konferenz in Puebla nicht als Arbeitsgrundlage diente, so macht dieser Hinweis aber doch einen wichtigen Schritt der lateinamerikanischen Kirche deutlich: Die Einbeziehung der Kommunikationsmittel in den Pastoralplan und die Deutung der Evangelisierung als Kommunikationsprozeß haben die Möglichkeit einer theologischen Integrierung und einer theologischen Aufarbeitung der Fragen der Massenmedien eröffnet. 


\section{Anmerkungen}

1 Vgl. dazu Piero Gheddo: In quale ambiente si è svolta la Conferenza di Puebla, in „Mondo e Missione” 108 (1979) 213-227, hier 216-218; Hans-Jürgen Prien, Puebla '79: Evangelisation Befreiung für den ganzen Menschen, Bilanz und Zukunft der katholischen Kirche Lateinamerikas, in: „der überblick” 15 (1979) 4-22, hier $18 \mathrm{f}$.

${ }^{2}$ Ferdinand Klostermann: Die Ungereimtheiten und Mißverständnisse von Puebla - ein kirchliches Kommunikationsproblem?, in: „Diakonia” 10 (1979) 186-189, hier $188 \mathrm{f}$.

${ }^{3}$ III Conferencia General del Episcopado Latinoamericano, La Evangelización en el Presente y en el Futuro de America Latina, Redacción provisional, Puebla de los Angeles, 27 de Enero 13 de Febrero de 1979, Nr. 30; weiterhin zitiert als Dok. Nr. . .; die deutsche Übersetzung und der offizielle Text des Hauptabschnitts über die Massenmedien im Puebla-Dokument vgl. CS 12: 1979, 289-296; diese wichtigen und klaren Aussagen über die Bedeutung und die Funktion der Kommunikation innerhalb der menschlichen Gemeinschaft und innerhalb der kirchlichen Wirklichkeit lassen das Verhalten gegenüber der Presse und Öffentlichkeit in Puebla noch unverständlicher erscheinen.

4 Dok. Nr. 421.

5 Dok. Nr. 716.

6 Dok. Nr. 934.

7 Dok. Nr. 959.

8 Dok. Nr. 1002.

9 Dok. Nr. 629.

10 Dok. Nr. 646.

11 Dok. Nr. 709.

12 Dok. Nr. 188.

13 Dok. Nr. 865.

14 Dok. Nr. 866.

15 Dok. Nr. 867

16 Dok. Nr. 868.

17 „Communio et Progressio”, in: AAS 63 (1971) 594-656; dazu vgl. auch Giselbert Deussen: Die Pastoralinstruktion auf dem Hintergrund der päpstlichen Lehrtradition, in: CS 4 (1971) 310-321; Michael Schmolke: Zehn ideengeschichtliche Beobachtungen zur Pastoralinstruktion „Communio et Progressio”, a.a.O. 299-309; Karl-Werner Bühler: Katholische Pastoralinstruktion und protestantische Uppsala-Erklärung zur Massenkommunikation, in: CS 5 (1972) 1-6; Franz-Josef Eilers: Die Pastoralinstruktion im Spiegel der Presse, a.a.O. 37-44; ebd.: Die Pastoralinstruktion zur Kommunikation in Entwicklungs- und Missionsländern, a.a.O. 197213; Friedhelm Geller: Die verschiedensprachigen Ausgaben der Pastoralinstruktion: Ein Vergleich, a.a.0. 309-326; Alois Huter: Die Pastoralinstruktion „Communio et Progressio" und das neue Bild vom Menschen, a.a.O. 97-108; Jesús Iribarren: Pastoralinstruktion und katholische Berufsorganisationen, a.a.O. 7-16; Otto Kaspar: Die Forderungen der Pastoralinstruktion an Hierarchie und Klerus, a.a.O. 16-19; John W. Mole: Die Pastoralinstruktion „Communio et Progressio" und das Konzilsdekret „Inter Mirifica”, a.a.O. 108-117; Frans Oudejans: Die Pastoralinstruktion „Communio et Progressio” aus der Sicht eines Journalisten, a.a.O. 118-123. 
18 „Evangelii nuntiandi”, in: AAS 68 (1976); vgl. dazu auch: Horst Rzepkowski: Der Welt verpflichtet, Text und Kommentar zu „Evangelii nuntiandi”, St. Augustin 1976, 140-142; Matteo Ajassa: I mezzi della comunicazione sociale e la fide formativa del nostro tempo, in: L'Annuncio del Vangelo oggi, Commento all'Esortazione Apostolica di Paolo VI „Evangelii nuntiandi”, Roma 1977, 555-590.

19 Alex J. van Wyk: Kommunikatie as sendingsproblem, in: Sendingwetenskap Vandag, 104-196, hier 105, zitiert nach Hans-Werner Gensichen: Glaube für die Welt, Theologische Aspekte der Mission, Gütersloh 1971, 189.

20 Luciano M. Metzinger: El Tema de la Comunicación Social en Puebla, in: SAL-OCIC, No. 84, Marzo-Mayo 1979, 1-4.

21 III Conferencia General del Episcopado Latinoamericano, La Evangelización en el Presente y en el Futuro de America Latina, Preparación, Documento de Consulta a las Conferencias Episcopales, Puebla-Mexico 1978, Nr. 21.

22 EN Nr. 45.

23 Vgl. dazu Horst Bürkle: Missionstheologie, Stuttgart 1979, 83-96, wo er eine Zusammenfassung und Auswertung der Literatur zum Zusammenhang von Kommunikation und Evangelisierung bietet.

24 Documento de Consulta ... Nr. 1041-1049.

\section{SUMMARY}

This article is an attempt to examine what was said about Communications at the CELAM Conference held in Puebla in 1979. At that conference, participants attributed a decisive role to the media in the work of evangelisation. Because of this convistion there arise certain demands regarding pastoral work and the proclamation of the Gospel by the Church. The Church, therefore, should try to familiarise itself with mass media. In present socio-cultural changes, media plays an important role. The process of evangelisation is understood in the Publa document to be a communications process. Communications is then to be an integral part of pastoral planning. An examination of recent Church documents will demonstrate that with the integration of mass media into pastoral work and the process of evangelisation, an important step has been made towards a theological understanding of communications. 


\section{RÉSUMÉ}

L'article veut répondre à deux questions. En premier lieu, ce que la conférence de Puebla a dit sur les mass media. On donne une importance décisive aux moyens de communication sociaux en matière d'evangélisation et, de là, on a des exigences précises pour la pastorale et la proclamation religieuse. Elles doivent avoir de l'influence sur les mass media, mais aussi les rendre familier. Dans le remaniement socio-culturel actuel, on prête aux mass media une haute valeur d'emploi. L'évangélisation est comprise comme processus de communication. Ainsi, on exige l'intégration dans le plan pastoral dans son ensemble. Une comparaison de quelques documents ecclésiastiques montre qu'ici, avec l'intégration des mass media dans le plan postoral et avec la révélation comprise comme un processus de communication, on a osé un pas important en direction d'une interprétation théologique de la communication sociale.

\section{RESUMEN}

El estudio quiere responder a dos interrogantes. El primero, qué dijo la Conferencia de Puebla sobre los medios de comunicación social. Se concede a los mass media una decisiva inportancia para la evangelización y de ahí arrancan diversas exigencias para la pastoral y la evangelización. Deben influir en los mass media, pero también familiarizarse con ellos. Se concede a los mass media un alto valor en la actual transformación sociocultural. La evangelización se define como proceso de comunicación. Por ello se exige la integración de la comunicación social en el plan pastoral. Una comparación con algunos documentos eclesiales muestra que, con la incorporación de los mass media al plan pastoral y con la comprensión de la evangelización como proceso de comunicación social se está dando un paso importante hacia una interpretación teológica de la comunicación social. 